\title{
CBCT assessment of radicular volume loss after rapid maxillary expansion: A systematic review
}

\author{
Antonino Lo Giudice ${ }^{1,2}$, Cosimo Galletti ${ }^{3}$, Cosme Gay-Escoda ${ }^{3}$, Rosalia Leonardi ${ }^{1}$ \\ ${ }^{1}$ Department of Medical-Surgical Specialties - Section of Orthodontics, School of Dentistry, University of Catania, Azienda Os- \\ pedaliero - Universitaria "PoliclinicoVittorio Emanuele", Via S. Sofia, 78 - 95123 Catania, Italy \\ ${ }^{2}$ Department of Biomedical and Dental Sciences and Morphofunctional Imaging - Section of Orthodontics, School of Dentistry, \\ University of Messina, Policlinico Universitario "G. Martino", Via Consolare Valeria - 98123 Messina, Italy \\ ${ }^{3}$ Oral and Maxillo-facial Surgery Department. School of Dentistry, University of Barcelona. Campus de Bellvitge UB, L’Hospitalet \\ de Llobregat, Barcelona, Spain
}

Correspondence:

Oral and Maxillo-facial Surgery Department

School of Dentistry, University of Barcelona

Campus de Bellvitge UB, L'Hospitalet de Llobregat

Barcelona, Spain

cosimo88a@gmail.com

Lo Giudice A, Galletti C, Gay-Escoda C, Leonardi R. CBCT assessment of radicular volume loss after rapid maxillary expansion: A systematic review. J Clin Exp Dent. 2018;10(5):e484-94. http://www.medicinaoral.com/odo/volumenes/v10i5/jcedv10i5p484.pdf

Received: 18/02/2018 Accepted: 11/04/2018

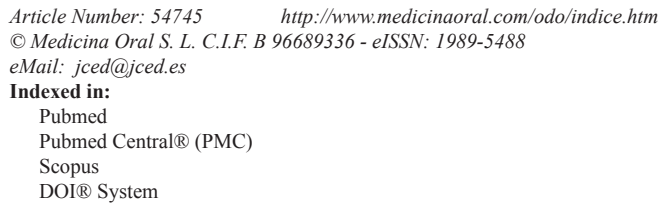

\begin{abstract}
Background: The present systematic review analyzed the current literature to investigate whether rapid maxillary expansion (RME) causes radicular resorption, assessed by cone-beam computed tomography (CBCT).

Data and Sources: Eighteen electronic databases and reference lists of studies were searched up to November 2017. Grey literature was also screened. To be included, articles must be human studies on growing subjects with transversal maxillary deficiency treated with maxillary expansion protocol and with 3-D radiographic assessment of radicular volume by CBCT images. Two authors independently performed study selection, data extraction, and risk of bias assessment. Study characteristics (study design, sample size, age, sex, skeletal maturity, type of appliance, daily activation, teeth evaluated, CBCT settings), and study outcomes (radicular volume loss) were reported according to the PRISMA statement.

Study selection: Only 3 articles were considered eligible and an individual analysis of the selected articles was undertaken. The risk of bias assessment revealed low methodological quality for all the studies included. In all the considered studies, significant radicular volume loss was observed in posterior teeth, following RME. When reported in percentage, the radicular volumetric loss was similar between anchored (first molars and first premolars) and unanchored teeth (second premolars).

Conclusions: A preliminary evaluation of the patient-related risk factors for RR is warmly advisable when administering RME.
\end{abstract}

Key words: RME, maxillary expansion, root resorption, external root resorption. 


\section{Introduction}

Root resorption (RR) represents a physiologic or pathologic condition resulting in the dissolution of cementum and dentin of dental roots (1). It represents an inevitable side effect of the orthodontic treatment with individual predisposition and orthodontic mechanics playing a role as etiological factors (2). However, the implication of $\mathrm{RR}$ in terms of viability and physiologic function of the involved teeth is still unclear (3).

Rapid maxillary expansion (RME) is the standard treatment for the correction of transversal maxillary deficiency $(4,5)$ in growing subjects. During the active phase, heavy forces are transmitted to the maxilla by the anchored teeth. Those forces determine the hyalinization of periodontal ligament, preventing dental movement (6). Later, with the permanence of residual forces stored in the appliance, the orthodontic effect initiates and the sequelae of periodontal ligament hyalinization may occur, i.e., buccal bone plate and $\operatorname{RR}(7,8)$.

Nevertheless, the diagnosis of RR is difficult due to the lack of clinical pathognomonic symptoms. During orthodontic treatment, indeed, clinicians may notice RR through routine examinations such as panoramic or periapical radiographs (9). However, conventional 2-D radiography presents large inaccuracies in detecting RR, especially when it is located at the buccal or lingual root surface (10) as it may occur after RME.

Within the last few years, cone-beam computed tomography $(\mathrm{CBCT})$ has been widely used in dentistry. It provides 3-D images of dental structures without projection errors and with less artifacts compared to conventional CT (11). CBCT has greater accuracy in detecting in-vitro simulated resorption cavities compared to conventional periapical radiographs (9). Furthermore, CBCT allows detecting radicular volume changes (12). Since RR is a kind of dental volume loss, the assessment of radicular volume is superior compared to a bidimensional analysis of root length (12).

In the present study, we systematically reviewed, for the first time, the bibliographic data from studies investigating RR following RME therapy by means of CBCT radiography in young patients.

\section{Material and Methods}

The present systematic review is consistent with the guidelines of the Cochrane Handbook for Systematic Reviews of Interventions (version 5.1.0) and is reported according to the PRISMA statement (13-15).

Two authors (A.L.G. and C.G.) independently carried out the selection of the studies, data collection and the assessment of risk of bias. Any disagreement was resolved by discussion with a third author (C.G.E.). This study was registered in the National Institute of Health Research database with an appropriate protocol number (http://www.crd.york.ac.uk/PROSPERO Protocol: CRD42016052064).
-Search Method

Searches were conducted on several electronic databases to find out articles concerning the effects of no-surgical skeletal maxillary expansion on dental root resorption, published up November 2017. Specific electronic databases were also searched for conference abstracts, dissertations, conference proceedings and unpublished literature (grey literature). The strategy search was adjusted for each database and is reported in Table 1, 1 continue, 1 continue- 1 . The reference lists of the articles eligible for inclusion were also manually reviewed. No restriction was applied to language, publication year, or status. Finally, authors were contacted to obtain specific data or info not provided in their article.

-Selection of studies

According to the PICOS (population, intervention, comparison, outcome, study design) format, the following inclusion criteria were selected to assess the eligibility of the studies: related human clinical studies; studies conducted on growing patients with maxillary transverse deficiency (Population); non-surgical skeletal maxillary expansion therapy (Intervention); control group represented by pre-treatment radicular volumetric measurements of posterior permanent teeth (Comparison) assessed by CBCT; post-treatment radicular volume loss of the same teeth (Outcomes measured); randomized or non-randomized clinical trials, cohort studies, case-control and retrospective studies (Study design). Studies including patients with previous orthodontic treatment, periodontal diseases, endodontic treatment of posterior teeth, tooth agenesis or with anomalies in form, shape, or structured and congenital syndromes were excluded. In a first phase, the authors screened all titles and abstracts retrieved from the databases and selected the studies that assessed radiographically root resorption after maxillary expansion. The reference lists of the studies included in phase 1 were also screened to retrieve additional eligible articles. In a second phase, the same authors reviewed the full texts of the studies selected in phase 1 and considered eligible only those assessing volumetric root resorption by means of CBCT images. The level of agreement between the two reviewers was assessed by the Cohen's kappa statistics.

-Data collection

Data extraction form was developed to collect the characteristics (study design, sample size, age, sex, skeletal maturity, type of appliance, daily activation, teeth evaluated, observation period, CBCT settings) and the outcomes (radicular volume loss) of the included studies. The Cohen's kappa statistics was performed to assess the agreement between the two authors.

-Assessment of risk of bias

Risk of bias assessment was performed using the Downs and Black scale 16 as suggested by the Cochrane Handbook for Systematic Reviews of Interventions (13-15) 
Table 1: Strategy searches used for the eighteen electronic databases.

\begin{tabular}{|c|c|c|}
\hline Database of Published Trials & Search strategy used & Hits \\
\hline $\begin{array}{l}\text { MEDLINE searched } \\
\text { Via PubMed } \\
\text { searched on November 5, } 2017 \\
\text { via www.ncbi.nlm.nih.gov/sites/entrez/ }\end{array}$ & $\begin{array}{l}((((()(((((\text { palatal expansion techniques[MeSH Terms]) OR maxillary } \\
\text { expansion[Title/Abstract]) OR rapid maxillary } \\
\text { expansion[Title/Abstract]) OR transverse maxillary } \\
\text { expansion[Title/Abstract]) OR maxillary transverse } \\
\text { deficiency[Title/Abstract]) OR SME[Title/Abstract]) OR } \\
\text { RME[Title/Abstract]) OR RPE[Title/Abstract]) OR } \\
\text { SPE[Title/Abstract])) AND (((((tooth resorption[MeSH Terms]) OR } \\
\text { root resorption[Title/Abstract]) OR apical root } \\
\text { resorption[Title/Abstract]) OR external root } \\
\text { resorption[Title/Abstract]) OR EARR))) NOT ((animals [mh] not } \\
\text { (humans [mh] and animals [mh]))) }\end{array}$ & 53 \\
\hline $\begin{array}{l}\text { OvidSP } \\
\text { searched on November 5, } 2017 \\
\text { via https://ovidsp.tx.ovid.com/ }\end{array}$ & $\begin{array}{l}\text { (maxillary expansion or rapid maxillary expansion or transverse } \\
\text { maxillary expansion or maxillary transverse deficiency or SME or } \\
\text { RME or RPE or SPE) AND (root resorption or apical root resorption } \\
\text { or external root resorption or EARR) }\end{array}$ & 114 \\
\hline $\begin{array}{l}\text { EMBASE searched via ScienceDirect } \\
\text { searched on November } 5,2017 \\
\text { via www.embase.com }\end{array}$ & $\begin{array}{c}\text { (maxillary expansion OR rapid maxillary expansion OR transverse } \\
\text { maxillary expansion OR maxillary transverse deficiency OR SME } \\
\text { OR RME OR RPE OR SPE) AND (root resorption OR apical root } \\
\text { resorption OR external root resorption OR EARR) }\end{array}$ & 529 \\
\hline $\begin{array}{l}\text { Cochrane Database of Systematic Reviews } \\
\text { searched via The Cochrane Library } \\
\text { searched on November 5, } 2017 \\
\text { www.thecochranelibrary.com }\end{array}$ & $\begin{array}{l}\text { ((maxillary expansion) OR (rapid maxillary expansion) OR } \\
\text { (transverse maxillary expansion) OR (maxillary transverse } \\
\text { deficiency) OR (SME) OR (RME) OR (RPE) OR (SPE)) AND ((root } \\
\text { resorption) OR (apical root resorption) OR (external root resorption) } \\
\text { OR (EARR)) }\end{array}$ & 0 \\
\hline $\begin{array}{l}\text { Cochrane Central Register of Controlled } \\
\text { Trials searched via The Cochrane Library } \\
\text { searched on November } 5,2017 \\
\text { www.thecochranelibrary.com }\end{array}$ & $\begin{array}{l}\text { ((maxillary expansion) OR (rapid maxillary expansion) OR } \\
\text { (transverse maxillary expansion) OR (maxillary transverse } \\
\text { deficiency) OR (SME) OR (RME) OR (RPE) OR (SPE)) AND ((root } \\
\text { resorption) OR (apical root resorption) OR (external root resorption) } \\
\text { OR (EARR)) }\end{array}$ & 4 \\
\hline $\begin{array}{l}\text { Google Scholar } \\
\text { searched on November 5, } 2017 \text { via } \\
\text { www.scholar.google.com }\end{array}$ & $\begin{array}{l}\text { (“maxillary transverse deficiency" OR “maxillary expansion” OR } \\
\text { "rapid maxillary expansion” OR “transverse maxillary expansion” } \\
\text { OR SME OR RME OR RPE OR SPE) AND (“root resorption” OR } \\
\text { “apical root resorption” OR “dental resorption” OR EARR) }\end{array}$ & 980 \\
\hline
\end{tabular}


Table 1 continue: Strategy searches used for the eighteen electronic databases.

\begin{tabular}{|c|c|c|}
\hline $\begin{array}{l}\text { Web of Science } \\
\text { searched on November 5, } 2017 \\
\text { via www.webofknowledge.com }\end{array}$ & $\begin{array}{l}\text { (maxillary expansion or rapid maxillary expansion or transverse } \\
\text { maxillary expansion or maxillary transverse deficiency or SME or } \\
\text { RME or RPE or SPE) } \\
\text { AND } \\
\text { (root resorption or apical root resorption or external root resorption } \\
\text { or EARR) }\end{array}$ & 89 \\
\hline $\begin{array}{l}\text { Scopus } \\
\text { searched on July 27, } 2017 \\
\text { via www.scopus.com }\end{array}$ & $\begin{array}{l}\text { (maxillary expansion OR rapid maxillary expansion OR transverse } \\
\text { maxillary expansion OR maxillary transverse deficiency OR SME } \\
\text { OR RME OR RPE OR SPE) AND (root resorption OR apical root } \\
\text { resorption OR external root resorption OR EARR) }\end{array}$ & 129 \\
\hline $\begin{array}{l}\text { African Journal Online Database } \\
\text { searched on July } 27,2017 \\
\text { via www.ajol.info }\end{array}$ & $\begin{array}{l}\text { (Maxillary expansion) AND ((root resorption) OR (dental } \\
\text { resportion)) }\end{array}$ & 0 \\
\hline $\begin{array}{l}\text { LILACS } \\
\text { searched on July } 27,2017 \\
\text { via http://bvsalud.org/en/ }\end{array}$ & $\begin{array}{c}\text { (tw:(maxillary expansion)) AND (tw:(dental resorption) OR tw: (root } \\
\text { resorption)) }\end{array}$ & 185 \\
\hline $\begin{array}{l}\text { Bandolier } \\
\text { searched on July } 27,2017 \text { via } \\
\text { http://www.medicine.ox.ac.uk/bandolier/ }\end{array}$ & (Maxillary expansion) AND (root resorption) & 0 \\
\hline \multicolumn{3}{|c|}{ Databases of dissertations and conference proceedings } \\
\hline $\begin{array}{lr}\text { Digital dissertation } & \\
\text { searched via UMI ProQuest } & \text { searched on } \\
\text { July } \quad 30, \quad 2017 & \text { via } \\
\text { http://search.proquest.com/pqdtft/dissertations/fromB } \\
\text { asicHomePage }\end{array}$ & $\begin{array}{c}\text { (maxillary expansion or rapid maxillary expansion or transverse } \\
\text { maxillary expansion or maxillary transverse deficiency or SME or } \\
\text { RME or RPE or SPE) AND (root resorption or apical root resorption } \\
\text { or external root resorption or EARR) }\end{array}$ & 0 \\
\hline $\begin{array}{l}\text { Conference Proceedings Citation Index searched on } \\
\text { July 30, } 2017 \text { via Web of W } \\
\text { Science,http:/thomsonreuters.com/conference- } \\
\text { proceedings-citation-index/ }\end{array}$ & $\begin{array}{l}\text { (maxillary expansion or rapid maxillary expansion or transverse } \\
\text { maxillary expansion or maxillary transverse deficiency or SME or } \\
\text { RME or RPE or SPE) AND (root resorption or apical root resorption } \\
\text { or external root resorption or EARR) }\end{array}$ & 1 \\
\hline \multicolumn{3}{|l|}{ Databases of research registers } \\
\hline $\begin{array}{l}\text { German Library of Medicine (ZB Med) searched on } \\
\text { July 30, } 2017 \text { via http://www.medpilot.de }\end{array}$ & $\begin{array}{l}\text { (maxillary expansion OR rapid maxillary expansion OR transverse } \\
\text { maxillary expansion OR maxillary transverse deficiency OR SME } \\
\text { OR RME OR RPE OR SPE) AND (root resorption OR apical root } \\
\text { resorption OR external root resorption OR EARR) }\end{array}$ & 100 \\
\hline
\end{tabular}


Table 1 continue-1: Strategy searches used for the eighteen electronic databases.

\begin{tabular}{|l|c|c|}
\hline $\begin{array}{l}\text { Metaregister of Controlled Clinical Trials searched } \\
\text { on July 30, 2017 via www.controlled-trials.com }\end{array}$ & $\begin{array}{l}\text { (maxillary expansion OR rapid maxillary expansion OR transverse } \\
\text { maxillary expansion OR maxillary transverse deficiency OR SME } \\
\text { OR RME OR RPE OR SPE) AND (root resorption OR apical root } \\
\text { resorption OR external root resorption OR EARR) }\end{array}$ & 0 \\
\hline $\begin{array}{l}\text { Clinical Trials.Gov } \\
\text { searched on July 30, 2017 via } \\
\text { http://clinicaltrials.gov/ct2/home }\end{array}$ & $\begin{array}{l}\text { (maxillary expansion OR rapid maxillary expansion OR transverse } \\
\text { maxillary expansion OR maxillary transverse deficiency OR SME } \\
\text { OR RME OR RPE OR SPE) AND (root resorption OR apical root } \\
\text { resorption OR external root resorption OR EARR) }\end{array}$ & 0 \\
\hline $\begin{array}{l}\text { "International Clinical Trials Registry Platform } \\
\text { searched on July 30, 2017 via } \\
\text { http://www.who.int/ictrp/en/" }\end{array}$ & $\begin{array}{c}\text { (maxillary expansion OR rapid maxillary expansion OR transverse } \\
\text { maxillary expansion OR maxillary transverse deficiency OR SME } \\
\text { OR RME OR RPE OR SPE) AND (root resorption OR apical root }\end{array}$ & 2 \\
\hline & resorption OR external root resorption OR EARR) & $\mathbf{2 1 8 6}$ \\
\hline
\end{tabular}

$\mathrm{SME}=$ slow maxillary expansion; $\mathrm{RME}=$ rapid maxillary expansion; $\mathrm{RPE}=$ rapid palatal expansion; $\mathrm{SPE}=$ slow palatal expansion; $\mathrm{EARR}=$ external apical root resorption.

The instrument consisted of 27 questions evaluating (1) reporting [10 questions], (2) external validity [3 questions], (3) internal validity or bias [7 questions], (4) internal validity or confounding or selection bias [6 questions], and (5) power [1 question]. According to this scale, answers were scored from 0 to 1 point, except for 2 items: reporting domain (question number 5) scored from 0 to 2 points and power analysis (last question) scored from 0 to 5 points. Consequently, the total maximum score that a clinical trial could receive was 32 points. The level of agreement between the two review authors was assessed with the Cohen's kappa statistic.

-Data analysis

Meta-analysis of the extracted data would have been considered only if the methodology was consistent among the included studies and if they reported equivalent volumetric measurements.

\section{Results and Discussion}

-Selection of studies

2186 articles were initially identified and 1636 remained after duplicates' removal; a total of 1629 articles were excluded after the reading of titles and abstracts and the full texts of the remained 7 articles were retrieved (Phase 1) (17-23). After reading the full texts of those studies, only 3 articles were considered eligible for the final inclusion in the present systematic review (Phase 2) (17-19).

Figure 1 shows the flow diagram for the selection of studies, and the excluded articles alongside with the reasons for their exclusions in both Phase 1 and Phase 2 are shown respectively in Table 2 and Table 3 . The agree- ment between the reviewers was highly reliable, with a kappa value of 0.984 .

-Study characteristics

The characteristics of the three studies included in this systematic review are summarized in Table 4. Two studies reported a retrospective cohort $(17,18)$ and one was designed as prospective study (19) All the selected articles investigated the radicular volume loss of posterior teeth after skeletal maxillary expansion by CBCT. All studies took place in a university setting and included both male and female participants. The mean age of the patients was almost identical in the three studies: 12.8 years $(17,19)$ and 12.7 years $(18)$. Only one study presented a control group and was constituted by the corresponding mandibular teeth (17). The following types of maxillary expander were used: Hyrax expander with bands on the first premolars and first molars $(18,19)$. Hyrax expander with extended arms to the second and first premolars (17) and Haas expander with bands on the first premolars and first molars (19). The protocol of expansion ranged from $0.25 \mathrm{~mm} 17$ to 0.50 mm18,19 of daily activation. One study (19) reported data of skeletal maturity that were based on hand-wrist radiograph. The timing of radiological assessment was different among the three studies: immediately after the active treatment phase $(18,19)$ and after 4.8 months of appliance retention; (17) moreover, one study (19) reported data of follow-up after six months of appliance retention. According to the design of the appliances tested, two investigations $(18,19)$ assessed the radicular volume loss of first molars, first premolars (anchored teeth) and second premolars (unanchored teeth), whereas one 


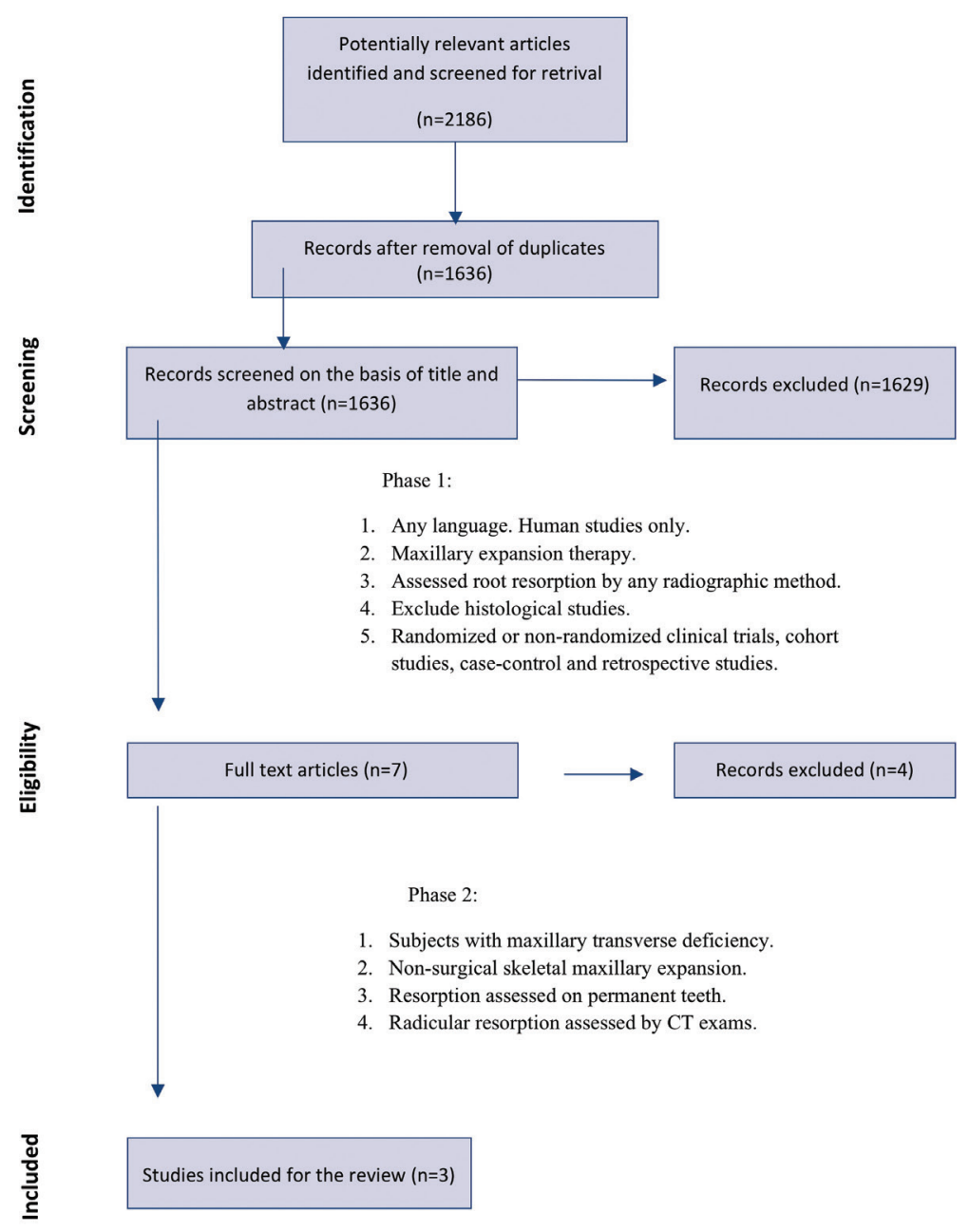

Fig. 1: Flow chart of study selection performed according to the PRISMA guidelines.

Table 2: Excluded articles with reason for exclusion during phase 1 of studies selection.

\begin{tabular}{|l|c|}
\hline Reason for exclusion & Excluded Articles \\
\hline Topic not compatible with the subject of the study & 818 \\
\hline Replies to authors or editor & 5 \\
\hline Interviews & 166 \\
\hline Book, conference abstracts or thesis & 53 \\
\hline Summaries or author index & 83 \\
\hline Reviews & 8 \\
\hline Meta-analysis & 88 \\
\hline Animal Studies & 11 \\
\hline In-vitro studies & 78 \\
\hline Studies on molecular biology, histology or genetics & 216 \\
\hline Surgical studies & $\mathbf{1 5 3 4}$ \\
\hline Total & \\
\hline
\end{tabular}


Table 3: Excluded articles with reason for exclusion during phase 2 of studies selection.

\begin{tabular}{|c|c|}
\hline Studies & Reason for exclusion \\
\hline Silva Filho et al. (2009) (20) & The study evaluated root resorption of deciduous teeth. \\
\hline Silva Filho et al. (1994) (21) & The study evaluated root resorption of posterior teeth with 2D radiography \\
\hline Jensen et al. (2015) (22) & The study employed surgically assisted rapid maxillary expansion. \\
\hline Kayalar et al. (2015) (23) & The study employed surgically assisted rapid maxillary expansion. \\
\hline
\end{tabular}

study (17) analyzed the first molars (anchored teeth) and the first premolars (wire-supported teeth). All CBCT images were at high resolution with different exposure parameters and with a reported voxel size of $0.18 \mathrm{~mm}$ (17), $0.20 \mathrm{~mm}$ (19) and $0.30 \mathrm{~mm}$ (18). The methodology and the volumetric measurements were heterogeneous among the three investigations: one study (18) measured the volume of each root of first molars, while two studies $(17,19)$ measured the absolute radicular volume. In two studies $(17,18)$ the roots were separated over the cement-enamel junction due to the presence of bands on molar teeth, and one study (20) included the cervical region in the assessment of radicular volume. The agreement between reviewers was highly reliable with a kappa value of 0.997 .

-Assessment of risk of bias

The overall score for the assessment of the risk of bias for each included studies (17-19) was evaluated as low, indicating poor reporting and experimental design in accordance with to the Downs and Black scale (Table 5) (16). The agreement between reviewers was highly reliable with a kappa value of 0.904 .

-Data analysis

A meta-analysis was not feasible due to the heterogeneity in study designs and the absence of comparable volumetric measurements among the included studies. Consequently, the risk of bias across the studies could not be performed and a descriptive analysis of the reported results was conducted.

To the best of our knowledge, this is the first systematic review investigating the existing literature in order to evaluate whether RME therapy causes the RR of posterior teeth, as assessed in vivo by CBCT. A previous systematic review has dealt with 2-D radiographic assessment of RR following RME, (24) however the authors reported that the literature was inconclusive due to the limitations of periapical radiographs to reveal RR, except for frank apical resorption.

We performed a wide and accurate bibliographic search and we found only 3 articles eligible for inclusion. The methodological quality of the included studies was limited, as assessed by the employed bias assessment tool. Also, these studies failed to give the higher level of scientific evidence, which is only attainable through the use of randomized clinical trials (25). Further prospective $3 \mathrm{D}$ studies, with adequate control groups, different retention periods and follow-ups are required in order to quantitatively assess RR in patients undergoing RME therapy. However, this is hardly possible due to the recommendations of the British Orthodontic Society and the American Association of Orthodontists concerning the use of $\mathrm{CBCT}$ and the risks related to ionizing radiation exposure for each patient (26-29). In the absence of the highest level of evidence, clinicians have to make decisions based on lower levels of evidence such those provided by the study included in the present systematic review.

A meta-analysis was not possible because the methodology and the reported information were heterogeneous among the included studies. Hence, we reported a descriptive analysis of the obtained results.

According to the findings of this systematic review, skeletal maxillary expansion induces radicular volume loss of posterior teeth. By comparing radicular volumes before and after RME, all the included studies (17-19) consistently reported the first molars as the teeth mostly affected by radicular resorption with mean values of volume loss ranging from $83.12 \mathrm{~mm}^{3}$ to $37.4 \mathrm{~mm}^{3}$. First premolars showed radicular volume loss ranging from $40.86 \mathrm{~mm}^{3}$ to $13.12 \mathrm{~mm}^{3}$ and second premolars from $37.64 \mathrm{~mm}^{3}$ to $13.93 \mathrm{~mm}^{3}$ (Table 4). Two studies $(18,19)$ reported also the mean percentage of radicular volume loss: for the first molars, Dindaroğlu et al. (19) found a total mean volume loss of $6.87 \%$, whereas Baysal et al. (18) reported the percentage of volume loss for each root i.e., $13.70 \%$ for the mesio-buccal root, $10.52 \%$ for the disto-buccal root and $10.50 \%$ for the palatal root. The mean percentage of volume loss ranged from $5.58 \%$ to $7.21 \%$ for the first premolars and from $5.77 \%$ to $6.47 \%$ for the second premolars. No statistically significant differences were found in the percentage of radicular volume loss among the teeth investigated $(18,19)$.

The extension of radicular resorption after RME can be influenced by the retention period and by the timing of radiographic examination. In this respect, the cumulating forces stored in the appliance and the relapse forces may sustain the resorption process even during the retention phase $(3,30)$. Meanwhile, the cementum repara- 


\begin{tabular}{|c|c|c|c|}
\hline 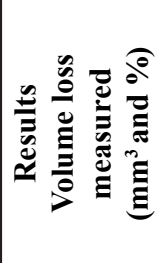 & 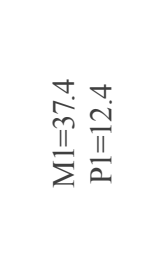 & 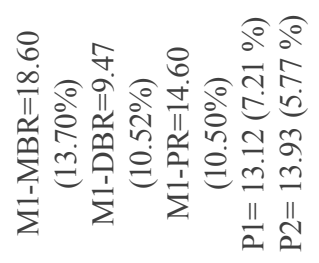 & 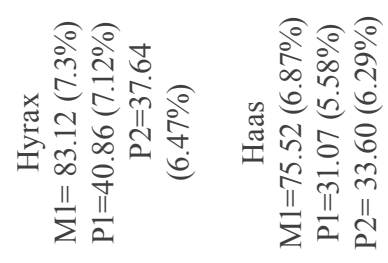 \\
\hline 已气 & 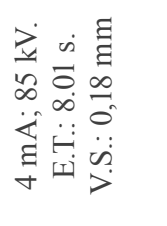 & 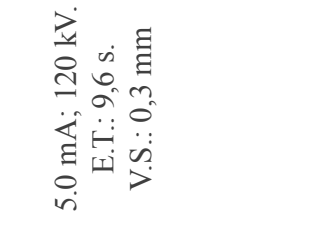 & 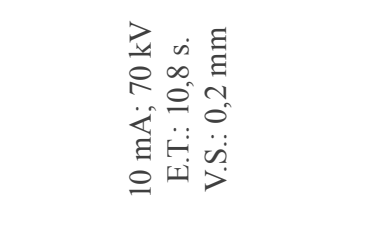 \\
\hline 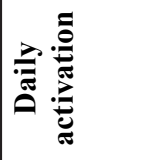 & $\begin{array}{c}\text { g } \\
\text { an } \\
\\
\text { o }\end{array}$ & $\begin{array}{l}\text { 音 } \\
\vdots \\
0 \\
0\end{array}$ & $\begin{array}{l}\text { E } \\
\text { ñ } \\
0\end{array}$ \\
\hline 莺 & $\bar{\Sigma} \bar{a}$ & $\bar{\Sigma} \bar{a} \cong$ & $\bar{\Sigma} \bar{a} \tilde{\Sigma}$ \\
\hline 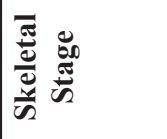 & ' & ' & 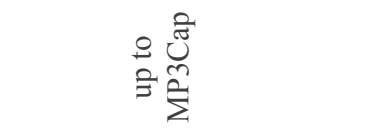 \\
\hline 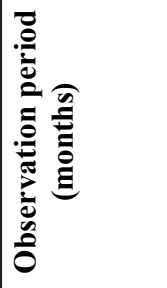 & 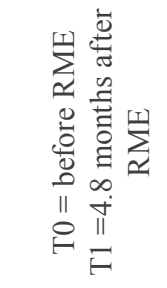 & 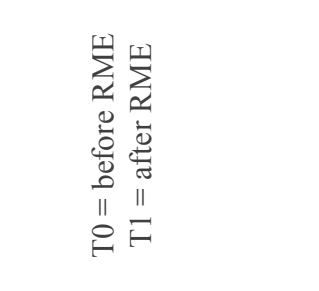 & 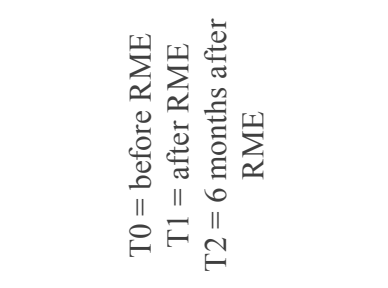 \\
\hline 苛 & 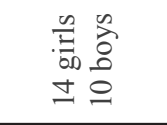 & 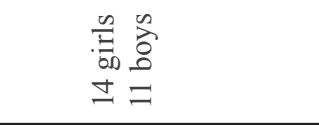 & 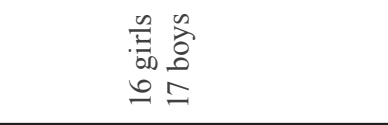 \\
\hline 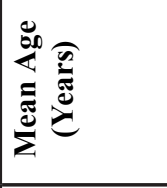 & $\begin{array}{l}\infty \\
\stackrel{\leftrightarrow}{\beth}\end{array}$ & $\hat{\mathrm{I}}$ & 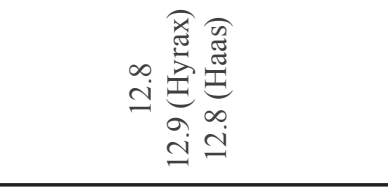 \\
\hline 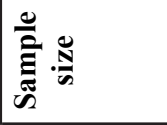 & 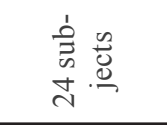 & 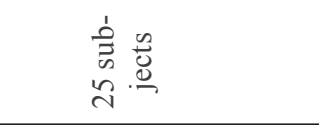 & 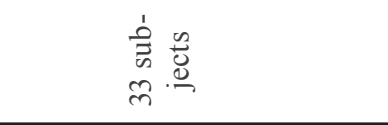 \\
\hline 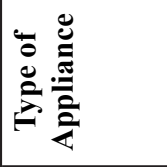 & 离 & 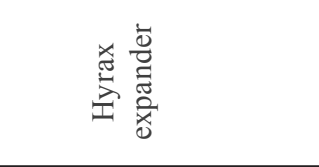 & 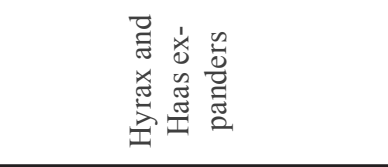 \\
\hline 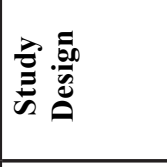 & 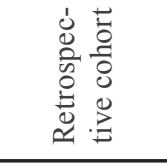 & 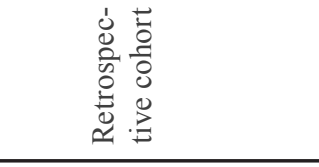 & 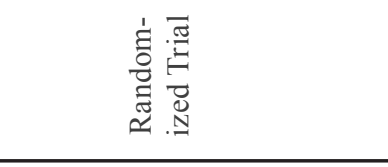 \\
\hline : & 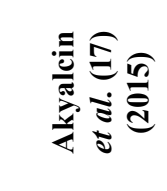 & 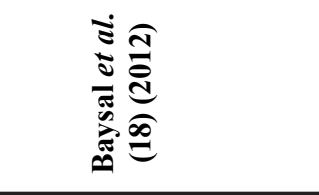 & 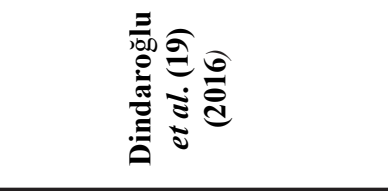 \\
\hline
\end{tabular}


Table 5: Risk of bias evaluation of selected studies.

\begin{tabular}{|c|c|c|c|c|c|c|}
\hline STUDIES & Reporting & $\begin{array}{c}\text { External } \\
\text { Validity }\end{array}$ & Bias & Confounding & Power & Overall \\
\cline { 2 - 6 } & $0-11$ & $0-3$ & $0-7$ & $0-6$ & $0-5$ & $0-32$ \\
\hline $\begin{array}{c}\text { Akyalcin } \text { et al. (17) } \\
(2015)\end{array}$ & 7 out of 11 & 2 out of 3 & 4 out of 7 & 1 out of 6 & 0 out of 5 & 14 out of 32 \\
\hline \begin{tabular}{c} 
Baysal et al. (18) (2012) \\
\hline $\begin{array}{c}\text { Dindaroğlu } \text { et al. (19) } \\
(2016)\end{array}$
\end{tabular} & 7 out of 11 & 2 out of 3 & 4 out of 7 & 1 out of 6 & 0 out of 5 & 14 out of 32 \\
\hline
\end{tabular}

tive process starts when the orthodontic forces are below a certain level (31). Thus, both processes may occur simultaneously during the retention period. In such cases, the resorption process may prevail over the cementum repair to an extent that may depend on several variables including patient age, skeletal maturity, subjective biological response and the protocol of maxillary expansion. This makes difficult to detect the true extension of RR (24). Among the considered articles, only Dindaroğlu et al. (19) reported a follow-up evaluation of radicular volumes after RME. The authors found a small radicular volumetric recovery that ranged from $4.43 \mathrm{~mm}^{3}$ for first premolars in the Hyrax group to $25.14 \mathrm{~mm}^{3}$ for first molars in the Haas group. Although cementum repair can only be assessed by histological analysis of radicular structural changes (8), it can be deduced that the volumetric differences of each tooth, between T1 (after expansion) and T2 (6 months retention period), reflect the amount of reparative cementum.19 Such volumetric recovery may be underestimated because CBCT cannot detect the repaired cementum until it is completely re-mineralized (31). These findings, however, would suggest that 6 months of retention may be insufficient to appreciate the full extension of tissue repair.

Patients' mean age was about 12.8 years in all the three studies (17-19). At these ages, the root development could not be fully completed, especially for second maxillary premolars. Thus, the volumetric data reported following RME therapy could be partially attributed to the disruption of roots' development. In this respect, Akyalcin et al. (17) found a slight increase in both volume and radicular length in the control group (mandibular first molars and premolars) compared to the tested group. However, no conclusion can be drawn due to the statistical and clinical irrelevance of the reported data and because such increment in the control group could be attributed to the continuous formation of cementum as consequence of changes in the occlusion (17).

It has been hypothesized that tissue borne appliances caused less radicular resorption than tooth-borne appliances due to the acrylic plate that distribute the expansion forces to supporting tissue.10 In this respect, Dindaroğlu et al. (19) found that the radicular volume loss was greater in the Hyrax group compared to the Haas group. However, such differences were found to be not statistically significant $\left(10.6 \mathrm{~mm}^{3}\right.$ for first molars, $9.79 \mathrm{~mm}^{3}$ for first premolars, and $4.04 \mathrm{~mm}^{3}$ for second premolars) are clinically irrelevant.

Clinicians often use a modified version of the original Hyrax and Haas appliances featuring a wire-supported anchorage system instead of bands on first premolars. A recent histological study, with split-mouth design, reported no significant differences of root resorption between the first premolars supported by the two anchorage systems.32 According to these findings, Akyalcin et al., (17) which employed a wire-supported anchorage of first premolars, reported significant radicular volume loss of these teeth $\left(12.4 \mathrm{~mm}^{3}\right)$. However, no comparison can be made with the findings of the two other studies, i.e. Baysal17 (13.12 $\mathrm{mm}^{3}$ ) and Dindaroğlu (19) (Hyrax group $=40.86 \mathrm{~mm}^{3}$; Haas group $=31.07 \mathrm{~mm}^{3}$ ), due to the heterogeneity in the methodology applied.

CBCT technology is reported to possess a good accuracy in assessing volumetric measurements of teeth and RR (12,33). However, such technology is affected by a systematic error consisting in the manual segmentation procedures that are influenced by voxel sizes, artifacts and quality of images $(12,33)$. In this respect, Wang et al. (12) reported that CBCT with a $125-\mu \mathrm{m}$ voxel size $(0.125 \mathrm{~mm})$ could identify cavities larger than $3.47 \mathrm{~mm}^{3}$ but failed to detect cavities smaller than $1.07 \mathrm{~mm}^{3}$ because of manual segmentation. In the studies included in this systematic review, the voxel resolutions were 0.30 $\mathrm{mm}$ (17) $0.20 \mathrm{~mm}$ (18) and $0.18 \mathrm{~mm}$ (17). Thus, it may be the case that small volumetric changes might not be discerned, determining their underestimation.

-Clinical implications

The implication of RR in terms of viability and physiologic function of the involved teeth is still unclear (25) Clinicians, however, are called to keep the risk of tissue damage as low as possible while performing therapeutic procedures. In this respect, we suggest to clinicians: 1) 
to perform an accurate anamnesis in order to identify the patient-related risk factors for root resorption $(3,34) 2)$ in mixed dentition, to use primary first molars as anchored teeth if they are steady, 3 ) to avoid RME in youngster insofar as higher forces are applied to the dentition due to the difficulty in opening the mid-palatal suture (35). In this case, clinicians may refer to surgically assisted expansion procedures or orthognathic surgery for the treatment of transversal maxillary deficiency. Moreover, when posterior teeth are at higher risk of periodontal damage and the amount of maxillary transversal deficiency does not justify such invasive surgical procedures, a bone-born palatal could represent a valid alternative (36).

\section{Conclusions}

- RME causes radicular volume loss of posterior teeth with the first molars as the teeth mostly affected by radicular resorption, as assessed by CBCT.

- When reported in percentage, the radicular volumetric loss was similar between anchored (first molars and first premolars) and unanchored teeth (second premolars).

- Clinicians must take into account such adverse effect when they choose to treat transversal maxillary deficiency using a palatal expander anchored to the permanent dentition.

\section{References}

1. Blake M. Woodside DG, Pharoah MJ. A radiographic comparison of apical root resorption after orthodontic treatment with the edgewise and Speed appliances. Am J Orthod Dentofacial Orthop. 1995;108:7684.

2. Brezniak N, Wasserstein A. Root resorption after orthodontic treatment: part 2. Literature review. Am J Orthod Dentofacial Orthop. 1993; 103:138-46

3. Weltman B, Vig KW, Fields HW, Shanker S, Kaizar EE. Root resorption associated with orthodontic tooth movement: a systematic review. Am J Orthod Dentofacial Orthop. 2010;137:462-76; discussion 412A.

4. Liu S, Xu T, Zou W. Effects of rapid maxillary expansion on the midpalatal suture: a systematic review. Eur J Orthod 2015;37:651-5.

5. Martina R, Cioffi I, Farella M, Leone P, Manzo P, Matarese G, Portelli M, Nucera R, Cordasco G. Transverse changes determined by rapid and slow maxillary expansion--a low-dose CT-based randomized controlled trial. Orthod Craniofac Res. 2012;15:159-68.

6. Zhou Y, Long H, Ye N, Xue J, Yang X, Liao L, Lai W. The effectiveness of non-surgical maxillary expansion: a meta-analysis. Eur $\mathrm{J}$ Orthod. 2014;36:233-42.

7. Lo Giudice A, Barbato E, Cosentino L, Ferraro CM, Leonardi R. Alveolar bone changes after rapid maxillary expansion with tooth-born appliances: a systematic review. Eur J Orthod 2017 doi: 10.1093/ejo/ cjx057

8. Langford SR, Sims MR. Root surface resorption, repair, and periodontal attachment following rapid maxillary expansion in man. Am J Orthod Dentofacial Orthop. 1982;81:108-15

9. Yi J, Sun Y, Li Y, Li C, Li X, Zhao Z. Cone-beam computed tomography versus periapical radiograph for diagnosing external root resorption: A systematic review and meta-analysis. Angle Orthod. 2017:87:328-337.

10. Westphalen VP, Gomes de Moraes I, Westphalen FH, Martins WD, Souza P.H. Conventional and digital radiographic methods in the detection of simulated external root resorptions: a comparative study. Dentomaxillofac Radiol. 2004;33:233-5.

11. Scarfe WC, Farman AG, Sukovic P. Clinical applications of co- ne-beam computed tomography in dental practice. J Can Dent Assoc. 2006;72:75-80.

12. Wang Y, He S, Guo Y, Wang S, Chen S. Accuracy of volumetric measurement of simulated root resorption lacunas based on cone beam computed tomography. Orthod Craniofacial Res. 2013;16:169-76.

13. Cochrane CollaborationIn: Higgins, J.P.T., Green, S., editors. Cochrane Handbook for Systematic Reviews of Interventions (version 5.1.0, updated March 2011). 2009: Available at: http://www. cochrane-handbook.org.

14. Liberati A, Altman DG, Tetzlaff J, Mulrow C, Gotzsche PC, Ioannidis JP, et al. The PRISMA statement for reporting systematic reviews and meta-analyses of studies that evaluate health care interventions: explanation and elaboration. J Clin Epidemiol. 2009;62:e1-34.

15. Nucera R, Lo Giudice A, Rustico L, Matarese G, Papadopoulos MA, Cordasco G. Effectiveness of orthodontic treatment with functional appliances on maxillary growth in the short term: a systematic review and meta-analysis. American Journal of Orthodontics and Dentofacial Orthopedics. 2016;149:600-611.

16. Downs SH, Black N. The feasibility of creating a checklist for the assessment of the methodological quality both of randomized and non-randomised studies of health care interventions. J Epidemiol Community Health. 1998;52:377-84.

17. Akyalcin S, Alexander SP, Silva RM, English JD. Evaluation of three-dimensional root surface changes and resorption following rapid maxillary expansion: a cone beam computed tomography investigation. Orthod Craniofacial Res. 2015;18:117-26.

18. Baysal A, Karadede I, Hekimoglu S, Ucar F, Ozer T, Veli I, et al. Evaluation of root resorption following rapid maxillary expansion using cone-beam computed tomography. Angle Orthod. 2012;82:488-94.

19. Dindaroğlu F, Doğan S. Evaluation and comparison of root resorption between tooth-borne and tooth-tissue borne rapid maxillary expansion appliances: A CBCT study. Angle Orthod. 2016;86:46-52.

20. da Silva Filho OG, Caldas R, Zambonato de Freitas P, Ferrari FM Jr. Influência da expansão rápida da maxila na rizólise dos caninos decíduos usados como ancoragem. Rev Dent Press Ortodon Ortop Facial. 2009;14:53-61.

21. da Silva Filho OG, Hernandes R, Okada T. Effects induced by rapid maxillary expansion on the anchorage bicuspids: radiographic study. Ortodontia. 1994;27:18-36.

22. Jensen T, Johannesen LH, Rodrigo-Domingo M. Periodontal changes after surgically assisted rapid maxillary expansion (SARME). Oral Maxillofac Surg. 2015;19:381-86.

23. Kayalar E, Schauseil M, Kuvat SV, Emekli U, Frratlı S. Comparison of tooth-borne and hybrid devices in surgically assisted rapid maxillary expansion: A randomized clinical cone-beam computed tomography study. J Craniomaxillofacial surg. 2016;44:285-93.

24. Forst D, Nijjar S, Khaled Y, Lagravere M, Flores-Mir C. Radiographic assessment of external root resorption associated with jackscrew-based maxillary expansion therapies: a systematic review. Eur J Orthod. 2014;36:576-85.

25. Lagravere MO, Major PW, Flores-Mir C. Long-term skeletal changes with rapid maxillary expansion: a systematic review. Angle Orthod. 2005;75:1046-52.

26. Isaacson K, Thom A, Horner K, Whaites E. Guidelines for the Use of Radiographs in Clinical Orthodontics. British Orthodontic Society. 2008. London, UK.

27. Halazonetis DJ. Cone-beam computed tomography is not the imaging technique of choice for comprehensive orthodontic assessment. Am J Orthod Dentofacial Orthop. 2012;141:403, 405, 407 passim.

28. Cordasco G, Portelli M, Militi A, Nucera R, Lo Giudice A, Gatto E, Lucchese A. Low-dose protocol of the spiral CT in orthodontics: comparative evaluation of entrance skin dose with traditional X-ray techniques, Prog Orthod. 2013;14:24.

29. Lo Giudice A, Fastuca R, Portelli M, Militi A, Bellocchio M, Spinuzza $P$, et al. Effects of rapid vs slow maxillary expansion on nasal cavity dimensions in growing subjects: a methodological and reproducibility study, Eur J Paediatr Dent. 2017;18:299-304.

30. Langford SR. Root resorption extremes resulting from clinical RME. Am J Orthod Dentofacial Orthop. 1982;81:371-77. 
31. Cheng LL, Türk T, Elekdağ-Türk S, Jones AS, Petocz P, Darendeliler MA. Physical properties of root cementum: Part13. Repair of root resorption 4 and 8 weeks after the application of continuous light and heavy forces for 4 weeks: a microcomputed-tomography study. Am J Orthod Dentofacial Orthop. 2009;136:320 e1-e10.

32. Martins DC, Souki BQ, Cheib PL, Silva GA, Reis ID, Oliveira DD, Nunes E. Rapid maxillary expansion: Do banded teeth develop more external root resorption than non-banded anchorage teeth? Angle Orthod. 2016;86:39-45

33. Liedke GS, da Silveira HE, da Silveira HL, Dutra V, de Figueiredo JA. Influence of voxel size in the diagnostic ability of cone beam tomography to evaluate simulated external root resorption. J Endod. 2009;35:233-35.

34. Elhaddaoui R, Benyahia H, Azeroual MF, Zaoui F, Razine R, Bahije L. Resorption of maxillary incisors after orthodontic treatment--clinical study of risk factors. Int Orthod 2016;14:48-64.

35. Melsen B. Palatal growth studied on human autopsy material. A histologic microradiographic study. Am J Orthod Dentofacial Orthop. 1975;68:42-54.

36. Lin L, Ahn HW, Kim SJ, Moon SC, Kim SH, Nelson G. Tooth-borne vs bone-borne rapid maxillary expanders in late adolescence, Angle Orthod. 2015;85:253-62.

\section{Conflicts of interest}

None to declaire. 